\title{
Frequency of Emerging Parasites in HIV/AIDS and Oncological Patients Stool by Coprological and Molecular Analysis
}

\author{
Enedina Jiménez-Cardoso ${ }^{1 *}$, Leticia Eligio-García ${ }^{1}$, Apolinar Cano-Estrada ${ }^{1}$, Adrian Cortés-Campos ${ }^{1}$, \\ Aurora Medina-Sansón ${ }^{1}$, Diana Molina-Martínez ${ }^{2}$
}

${ }^{1}$ Children Hospital of Mexico, Mexico City, Mexico; ${ }^{2}$ Specialized Clinic Condesa, Mexico City, Mexico.

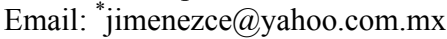

Received May $15^{\text {th }}, 2013$; revised June $15^{\text {th }}, 2013$; accepted July $15^{\text {th }}, 2013$

Copyright (C) 2013 Enedina Jiménez-Cardoso et al. This is an open access article distributed under the Creative Commons Attribution License, which permits unrestricted use, distribution, and reproduction in any medium, provided the original work is properly cited.

\begin{abstract}
The purpose of this study was to determine the frequency of emerging parasites in two groups of immunosuppressed patients, including individuals infected with the human immunodeficiency virus (AIDS) (HIV) or having acute lymphoblastic leukemia (ALL) with or without diarrhea. Stool samples were collected from 96 HIV and 77 ALL patients from March 2010 through December 2011. Screening for opportunistic parasites was carried out by the coproparasitoscopic Faust method, Ziehl Neelsen staining, and Polymerase Chain Reaction (PCR). Results showed that $22.9 \%$ of HIV fecal samples were positive for emerging parasites, including Cryptosporidium spp. (7.3\%), Microsporidium spp. (5.2\%), Isospora belli (1.0\%), Giardia intestinalis (2.6\%), and Cyclospora spp. (7.3\%). On the other hand, 32.5\% of ALL fecal samples were positive for emerging parasites, including Cryptosporidium spp. (9.1\%), Microsporidium spp. (19.5\%), Isospora belli (1.3\%), and Giardia intestinalis (2.6\%). Our results highlighted the need for specific, efficient, and reliable diagnostic methods to identify the presence of emerging parasites in immunocompromised patients susceptible to different infectious diseases or neoplastic processes and avoid the consequences for the host as an increased disease rate, alterations in the clinical manifestation of the infection or even exacerbation of its course.
\end{abstract}

Keywords: Leukemia; HIV; Emerging Parasites, Microsporidium; Cryptosporidium spp.; Giardia Intestinalis; Cyclospora; Isospora Belli

\section{Introduction}

Infections of the gastrointestinal tract are common in patients with acquired immunodeficiency syndrome (AIDS). Chronic diarrhea has been reported in up to $50 \%$ of the patients infected with the causative pathogen of AIDS, human immunodeficiency virus (HIV), in developed countries and up to $90 \%$ of those in developing countries [1]. This condition often results in considerable weight loss and dehydration that exacerbates the patient's already weakened physical condition and complicates the overburdened physiological processes. Several protozoa species have been associated with diarrhea in HIV-infected patients, including Cryptosporidium spp., Microsporidium spp., Isospora belli, Giardia intestinalis, and Cyclospora spp. [2]. Of these parasites, the Cryptosporidium and Microsporidium species are frequently

${ }^{*}$ Corresponding author. detected in the clinical samples and have been shown to preferentially cause diarrhea in patients with severely depressed peripheral blood $\mathrm{CD} 4^{+}$lymphocyte counts [3].

Only two members of the Cryptosporidium genus, $C$. parvum and C. hominis, infect humans [4]. These two species already represent an important public health threat, due to the fact that there is currently no curative antimicrobial therapy. As such, infection of immunocompromised individuals with these parasites is associated with a particularly high risk of fatality.

Intestinal microsporidiasis is most frequently caused by the Encephalitozoon and Enterocytozoon species [5]. The Microspora genus is composed of a broad group of single-celled obligate intracellular protozoa that share a polar filament and are capable of infecting evolutionarilydistinct hosts, from insects and fish to birds and mammals [6].

The coccidian Isospora belli, renamed Cystoisospora 
belli in 2005 has recently emerged as a medically important pathogen due to its prevalence in HIV patients. The incidence of $C$. belli infection in AIDS patients in the USA varies from $0.2 \%-3 \%$ and in Africa from $8 \%-$ $20 \%$; in addition, epidemiologic studies have revealed that isosporiasis may be related to travel and immigration from Latin America. This parasite causes a persistent diarrhea, malabsortion, and weight loss [7].

Giardia is a protozoan parasite found in the intestine of several mammals, including humans [8]. Giardia infections have been reported worldwide and have revealed a particularly high prevalence in children, HIV-positive individuals, and immunocompromised patients [9]. The symptoms of Giardia infection can range from asymptomatic to mild or severe malabsortion and chronic diarrhea. Moreover, the parasite is shed in the feces and transmitted by the fecal-oral route, and has a known risk of zoonotic transmission [10].

Cyclospora cayetanensis is a rapidly emerging parasite that has been implicated in both epidemic and endemic diarrheal illnesses around the world [11]. Several outbreaks of infection in industrialized countries, however, were found to be caused by contaminated produce imported from endemic areas. Human infection causes a wide range of symptoms in immunocompetent patients, the most frequent symptom will be mild to severe diarrhea [12]. Although, studies have shown that $C$. cayetanensis can be acquired orally and infect epithelial cells of the upper intestine [13]. While all of these parasitic infections can be passed directly from person-to-person, the most common route of transmission is believed to be ingestion of water or food contaminated by parasite-containing fecal matter. Worldwide study of these parasites' prevalence indicates a higher risk of infection in tropical and subtropical areas, and food products from these regions may be an important source of infection worldwide [14].

The antimicrobial therapies of choice for cyclosporiasis, cryptosporidiosis, and isosporiasis are trimethoprim and sulfamethoxazole [15], while the standard treatments for Giardiasis are metronidazole and albendazole [16]. For all, the effectiveness of therapy is variable and cases of resistance are common. Unfortunately, none of the currently available antimicrobials are efficacious for Enterocytozoon bieneusi, which is the most common microsporidian species identified in humans [17].

Traditionally, diagnosis of parasitic infection relies on microscopy-based observation and morphology-based identification of the organism in stool samples [18]. Alternative approaches have been recently developed, including immunological and molecular techniques based on advances in our knowledge of the physiological and genetic features of these emerging parasites [19]. Direct examination of fresh or concentrated feces remains the standard method of detection of I. belli and Giardia, since the large oocysts and cysts are readily visible by light microscopy without staining. However, microscopic identification of the smaller protozoan parasites is reliant upon the experience of the microscopist [20], since the morphological characteristics, size, and staining affinity are not uniform. Several studies have led to improved staining methods, and the newly developed molecular strategies have demonstrated high sensitivity and specificity for detecting and identifying the emerging human parasites. Therefore, a large scale screening of fecal samples from immunocompromised patients using these improved methods will provide more accurate insights into the prevalence and distribution of these medically important parasitic species.

The frequencies of emerging parasites, such as Cryptosporidium spp., in HIV patients in Mexico have been reported; however, the frequencies at which these emerging parasites are found in patients with other immunosuppressive diseases remains unknown. Despite their increasing presence in the HIV-positive population, the magnitude of the public health threat for these emerging parasites is undervalued. Other countries have reported on the frequency of opportunistic parasites in immunocompromissed patients, but the results have been variable, possibly due to insensitive methods used to detect and identify the infective species. The aim of this study was to determine the frequency and relationship of Cryptosporidium spp., Microsporidium spp., I. belli, Giardia intestinalis, and C. cayetanensis in fecal samples of immunocompromised patients with HIV or acute lymphoblastic leukemia (ALL) diagnoses, with and without gastrointestinal symptomatology, by using coproparasitoscopic and molecular methods.

\section{Methods}

\subsection{Fecal Specimen Collection from HIV and ALL Patients.}

From March 2010 through December 2011, we collected a total of 173 specimens of fecal material. Ninety-six of the samples were obtained from adult patients $(>17$ years old) with diagnosis of HIV who were being treated at the Specialized Clinic Condesa (Mexico City, Mexico). Seventy-seven of the samples were collected from pediatric patients $(<12$ years old $)$ with diagnosis of ALL who were being treated at the from Children's Hospital of Mexico (Mexico City, Mexico). The ALL fecal samples were collected at various disease stages, including time of diagnosis $(\mathrm{n}=23)$, second stage $(\mathrm{n}=18)$, third stage $(\mathrm{n}=16)$, and fourth stage $(\mathrm{n}=10)$. The study protocol was approved by the Institutional Ethics Committee of the Children's Hospital of Mexico. All adult patients 
provided written consent. For pediatric patients, written consent was provided by the parent or legal guardian.

\subsection{Coproparasitoscopic Analysis and Staining}

Fecal samples were stained with Lugol's iodine and examined under a light microscope in order to find cysts, oocysts and/or spores of parasites as previously described [21]. Liquid suspensions of fecal matter were stained with Ziehl Neelsen for viewing under an oil immersion lens as previously described [22]. Briefly, the fecal sample was fixed with methanol for $10 \mathrm{~min}$, rinsed with concentrated carbol-fuchsin for $20 \mathrm{~min}$, washed with 7\% sulfuric acid, and counterstained with Malachite green.

\subsection{PCR Amplification}

DNA was extracted from all fecal samples by use of the QIAamp ${ }^{\mathrm{TM}}$ DNA Stool Mini Kit (Qiagen Inc., Valencia, CA, USA) according to the manufacturer's instructions. The DNA concentrations were measured by a spectrophotometer (Epoch; Biotek, Winooski, VT, USA), then PCR was performed as described below.

Cryptosporidium spp. [23] were amplified from 500 ng of template DNA in a reaction volume of $25 \mu \mathrm{L}$, consisting of $1 \times$ PCR amplification buffer with dATP, dGTP, dCTP, and dTTP (1.2 $\mu \mathrm{M}$ each), $0.5 \mathrm{ng}$ each forward primer JVAF (5'-CCAATTACAAAACCAAAAAGTCC-3') and reverse primer JVAR (5'-ATGACGGGTAACGGGGAAT-3') and 1.5 U of Taq DNA polymerase (Roche, Mannheim, Germany). Thirty-five cycles of amplification were performed in a Maxi-gene thermal cycler (Axygen, Union City, CA, USA). After the initial denaturation of DNA at $94^{\circ} \mathrm{C}$ for $5 \mathrm{~min}$, each cycle consisted of a denaturation step at $94^{\circ} \mathrm{C}$ for $30 \mathrm{~s}$, an annealing step at $60^{\circ} \mathrm{C}$ for $30 \mathrm{~s}$, and an extension step at $72^{\circ} \mathrm{C}$ for $1 \mathrm{~min}$. A final extension step was carried out at $72^{\circ} \mathrm{C}$ for $10 \mathrm{~min}$.

To amplify the Microsporidium spp., the V1 (5'-CACCAGGTTGATTCTGCCTGAC-3') and PMP2 (5'-CCTCTCCGGAACCAAACCCTG-3') primers [24] were used in the above described $25 \mu \mathrm{L}$ reaction mixture. The thermal cycling conditions included initial denaturation of DNA at $95^{\circ} \mathrm{C}$ for $5 \mathrm{~min}$, followed by 35 cycles of denaturation at $94^{\circ} \mathrm{C}$ for $30 \mathrm{~s}$, an annealing step at $55^{\circ} \mathrm{C}$ for $30 \mathrm{~s}$, and an extension step at $72^{\circ} \mathrm{C}$ for $1 \mathrm{~min}$. A final extension step was carried out at $72^{\circ} \mathrm{C}$ for $10 \mathrm{~min}$.

Nested-PCR was used to amplify I. belli with the following primers [25]: outer primers, Iso-18SF0 (5'-CTGGTTGATCCTGCCAGTA-3') and Iso-28SR0 (5'-AAGGCTCAATCAAGAACCTCCG-3'); inner primers, Iso-18SF1 (5'-GATCCTGCCAGTAGTCAT-3') and Iso-28SR1 (5'-TGAAGCTAATCCCTCTTCC-3'). The reaction mix for both the first and second amplifica- tions was the same as described above. The thermal cycling conditions for both included initial denaturation of DNA at $95^{\circ} \mathrm{C}$ for $5 \mathrm{~min}$, followed by 35 cycles of denaturation at $94^{\circ} \mathrm{C}$ for $1 \mathrm{~min}$, an annealing step at $64^{\circ} \mathrm{C}$ for $1 \mathrm{~min}$, and an extension step at $72^{\circ} \mathrm{C}$ for $3 \mathrm{~min}$. A final extension step was carried out at $72^{\circ} \mathrm{C}$ for $10 \mathrm{~min}$.

For amplification of $G$. intestinalis [26], DNA the primers P (5'-GGTGGATCCTGCCGGAGCG-3') and A (5'-GCTCTCCGGAGTCGAAC-3') were used in the above described $25 \mu \mathrm{L}$ reaction mixture. The thermal cycling conditions included an initial denaturation of DNA at $97^{\circ} \mathrm{C}$ for $5 \mathrm{~min}$, followed by 35 cycles of denaturation at $94^{\circ} \mathrm{C}$ for $1 \mathrm{~min}$, an annealing step at $50^{\circ} \mathrm{C}$ for$1 \mathrm{~min}$, and an extension step at $72^{\circ} \mathrm{C}$ for $3 \mathrm{~min}$. A final extension step was carried out at $94^{\circ} \mathrm{C}$ for $5 \mathrm{~min}$.

Nested-PCR was used to amplify C. cayetanensis with the following primers [27]: outer primers, FIE (5'-TACCCAATGAAAACAGTTT-3') and R2B (5'-CAGGAGAAGCCAAGGTAGG-3'); inner primers, F3E (5'-CCTTCCGCGCTTCGCTGCGT-3') and R4B (5'-CGTCTTCAAACCCCCTACTG-3'). The reaction mix for both the first and second amplifications was the same as described above. The thermal cycling conditions for both reactions were similar, except that the $\mathrm{Tm}$ that was $53^{\circ} \mathrm{C}$ and $60^{\circ} \mathrm{C}$ for the first and second reaction, respectively. All PCR amplification products were electrophoretically resolved on a 2\% agarose gel (Promega, Madison, WI, USA) and stained with ethidium bromide for visualization under UV illumination.

\section{Results}

\subsection{HIV and ALL Patient Characteristics}

Of the 96 stool samples from the HIV adult patients, only five $(5.2 \%)$ were from females and 91 (94.8\%) were from males. Sixty of the HIV patients (62.5\%) had diarrhea at the time of sample collection. Of the 77 stool samples from the ALL pediatric patients, 29 (37.7\%) were from females and $48(62.3 \%)$ were from males. Only five of the ALL patients (6.4\%) had diarrhea at the time of sample collection (Tables 1(a) and (b)).

\subsection{Parasite Identification by Coproparasitoscopic Analysis and Staining}

Emerging parasites were detected in $22.9 \%$ (22/96) of the HIV patients, $59.1 \%(13 / 22)$ of whom had diarrhea. Among the 96 HIV patients, 7 were found to have Cryptosporidium, including 6 who had chronic diarrhea and one who had no diarrhea. Meanwhile, Microsporidium spp. was detected in 5 of the HIV patients, of which only one had diarrhea. I. belli was detected in only 1 HIV patient, and that patient had chronic diarrhea, and $G$. 
intestinalis was detected in only 2 HIV patients, of which only one had diarrhea. Finally, C. cayetanensis was detected in 7 of the HIV patients, 4 of whom had diarrhea and 3 of whom had no diarrhea (Table 1(a)). Emerging parasites were detected in $32.5 \%(25 / 77)$ of the ALL pediatric patients, but only 2 of those 25 had diarrhea. Of the 2 patients with diarrhea, 1 had Cryptosporidium and the other had Microsporidium (Table 1(b)). The total data are summarized in Tables 1(a) and (b) and show that Cryptosporidium and Cyclospora were more frequent in the HIV patients with diarrhea, while Cryptosporidium and Microsporidium were associated with diarrhea in the ALL patients. The rest of the studied parasites were detected at similar proportions between diarrhea and non-diarrhea cases.

Microscopic examination of parasites by the Faust coproparasitoscópico method allowed for morphology-based identification of Cryptosporidium spp., Microsporidium spp., I. belli, G. intestinalis, and C. cayetanensis (Figure 1). Figure 2 shows the frequency of emerging parasites in the HIV patients (panel A) and the ALL patients (panel B). Among the HIV adult patients,
Cryptosporidium spp. were detected in 7.3\% (7/96), Microsporidium spp. were detected in 5.2\% (5/96), I. belli was detected in $1.0 \%(1 / 96), G$. intestinalis was detected in $2.1 \%(2 / 96)$, and C. cayetanensis was found in $7.3 \%$ (7/96). Sixteen $(16.7 \%)$ of the HIV patient samples were identified as being infected with other microbes, including Escherichia coli, Entamoeba histolytica, Hymenolepis nana, and Iodameoba butschlii. In the ALL pediatric patients, Cryptosporidium spp. were detected in $9.1 \%$ (7/77), Microsporidium spp. were detected in $19.5 \%$ $(15 / 77)$, I. belli was detected in $1.3 \%(1 / 77)$, G. intestinalis was detected in $2.6 \%(2 / 77)$, and C. cayetanensis was not detected in any case. One of the patients with ALL was positive for Uncinaria.

Figure 3 shows the concordance of identification results between the coproparasitoscopic staining analyses (Faust CPS, Lugol-iodine, Ziehl Neelsen) and the molecular PCR analysis. The proportion of positive samples was greater with PCR than with the microscopic methods in both HIV and ALL patients. Thus, the molecular method was deemed to be more sensitive than the coproparasitoscopic methods, with staining-negative cases being PCR-positive.

Table 1. (a) Positive samples to parasites and its relation with diarrhea. Frequency of intestinal parasites in stool samples from HIV patients of Specialized Clinic Condesa; (b) Positive samples to parasites and its relation with diarrhea. Frequency of intestinal parasites in stool samples from ALL patients of Children Hospital of Mexico.

(a)

\begin{tabular}{|c|c|c|c|}
\hline \multicolumn{4}{|c|}{ Samples from HIV patients } \\
\hline$N=96$ & Female $=5 / 96(5.2 \%)$ & Male $=91 / 96(94.72$ & \\
\hline Parasite & With diarrhea $60 / 96(62.5 \%)$ & Without diarrhea 36/96 (37.5\%) & Total \\
\hline Cryptosporidium spp. & $6 / 7$ & $1 / 7$ & $7(7.29 \%)$ \\
\hline Microsporidium spp. & $1 / 5$ & $4 / 5$ & $5(5.2 \%)$ \\
\hline Isospora belli & $1 / 1$ & 0 & $1(1.04 \%)$ \\
\hline Giardia intestinalis & $1 / 2$ & $1 / 2$ & $2(2.08 \%)$ \\
\hline Cyclospora cayetanensis & $4 / 7$ & $3 / 7$ & $7(7.29 \%)$ \\
\hline Total emerging parasites & $13 / 96$ & 9/96 & $22(22.91 \%)$ \\
\hline $\begin{array}{c}\text { Other parasites (E. coli, E. histolytica, } \\
\text { H. nana or I. butschili) }\end{array}$ & 0 & 16 & $16(16.66 \%)$ \\
\hline
\end{tabular}

(b)

\begin{tabular}{cccc}
\hline & \multicolumn{2}{c}{ Samples from ALL patients } \\
\hline N = 77 & Female $=29 / 77(37.66 \%)$ & Male $=48 / 77(62.33 \%)$ & Total \\
\hline Parasite & With diarrhea $5 / 77(6.4 \%)$ & Without diarrhea $72 / 77(93.5 \%)$ & $7(9.09 \%)$ \\
\hline Cryptosporidium spp. & 1 & 6 & $15(19.48 \%)$ \\
Microsporidium spp. & 1 & 14 & $1(1.29 \%)$ \\
$\quad$ Isospora belli & 0 & 1 & $2(2.59 \%)$ \\
Giardia intestinalis & 0 & 2 & $\mathbf{2 5}(\mathbf{3 2 . 4 6 \% )}$ \\
Total emerging parasites & $\mathbf{2 / 7 7}$ & $\mathbf{2 3 / 7 7}$ & $1(1.29 \%)$ \\
Other parasites (Uncinaria) & 0 & 1 &
\end{tabular}




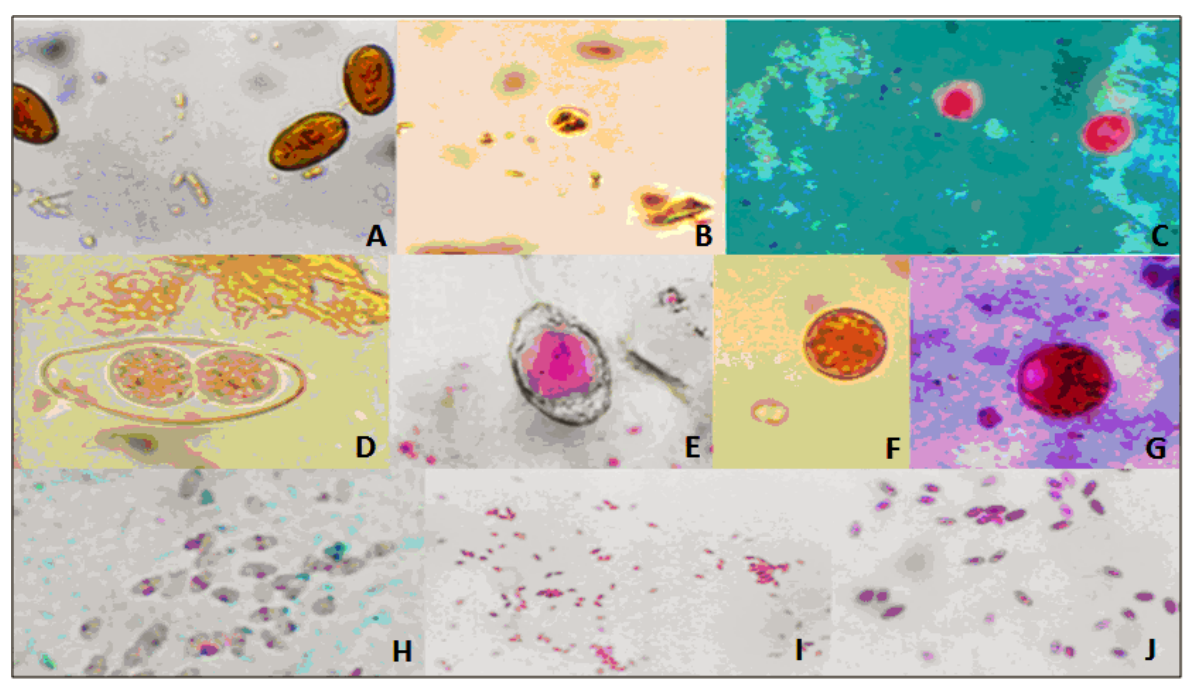

Figure 1. Morphology of parasites identified by light microscopy in stool samples of immunosuppressed patients under oil immersion is shown. (A) Cyst of Giardia intestinalis stained with lugol-Iodine (100×), we can observe the two nuclei characteristic of this specie; (B) Oocysts of Cryptosporidium spp., stained with Lugol-Iodine (100×) and (C) Cryptosporidium spp. oocysts, stained with Ziehl Neelsen method $(100 \times)$, the stain contribute to make a better identification; (D) Sporulated oocyst of Isospora belli showing two sporozoites $(100 \times)$ stained with Lugol-iodine; (E) Unsporulated Oocyst of Isospora belli (100×), stained with Ziehl Neelsen method. Because of its size this parasite can be identified even without a specialized staining; (F)-(G) Oocyst of Cyclospora cayetanensis (100×) stained with Lugol-Iodine (left) and with Ziehl Neelsen (right), the morphology of cyclospora is typical, but sometimes the identification is difficult because it have not a clear iodine affinity; (H)-( $\mathrm{J})$ Spores of two different preparations of Microsporidium spp. stained with Ziehl Neelsen method. $\mathrm{H}$ and $\mathrm{J}$ are seen at $100 \times$ and I at $40 \times$. It can't be observed only with lugol-iodine. The morphologic differences between $H$ and $J$ suggest the presence of two species.

\subsection{PCR Amplification}

The products of PCR are presented in Figure 4, we observed a 150-bp amplicon for Cryptosporidium spp., a 300-bp amplicon for Microsporidium spp., a 400-bp nested-PCR amplicon for I. belli, a 298 bp amplicon from $G$. intestinalis, and a 300-bp nested-PCR amplicon for $C$. cayetanensis.

\section{Discussion}

This work highlights the importance of knowing which are emerging and re-emerging parasites prevalent in immunosuppressed patients (HIV infected and diagnosed with ALL), in order to administer the best treatment and avoid additional pathologies that cause complications in addition to mmunosuppressive disease.

The distributions and clinical manifestations of each of the emerging pathogens were found to differ significantly between HIV patients and ALL patients. In the HIV patients, the parasites associated with diarrhea were Cryptosporidium spp. and C. cayetanensis, while in the ALL patients, the parasites associated with diarrhea were Cryptosporidium spp. and Microsporidium spp. These findings indicated that the presence of emerging parasites is not always associated with diarrhea in these two groups of patients. Diarrhea is one of the most common gastrointestinal symptoms in HIV-infected individuals and can be caused by a multitude of pathogens, including bacteria and viruses, or physiologic disruptions resulting from the stringent regimen of pharmacologic agents. Similarly, diarrhea in ALL patients is often the consequence of the chemotherapeutic drugs, which are known to affect tissues that require a high rate of cell multiplication, such as the intestinal mucosa, thereby producing diarrhea and promoting the colonization by different microorganisms.

Both groups in the current study were immunosuppressed; however, their compositions differed significantly in disease etiology (HIV infection and ALL cancer), sex (strong male bias among HIV patients), and age (adults and children), all of which may have different physiologic and molecular consequences on host-parasite interactions. The ALL patients were able to be stratified by duration of disease, which generally corresponded to duration of chemotherapeutic treatments. We noted that positive results for emerging pathogens were more frequent in the ALL patients who had received chemotherapy for at least nine months (data not shown). These patients are expected to have a more severely depressed immune system than the other ALL patients who have received less chemotherapy. However, we did not notice any trend in positive results for HIV patients according to 


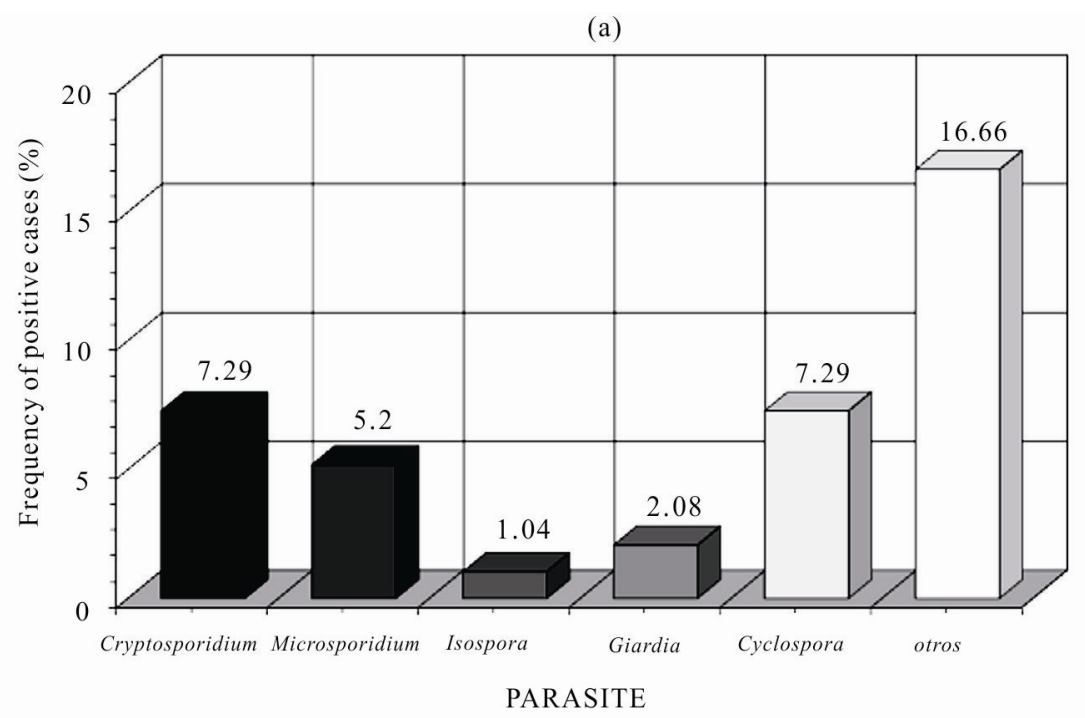

(b)

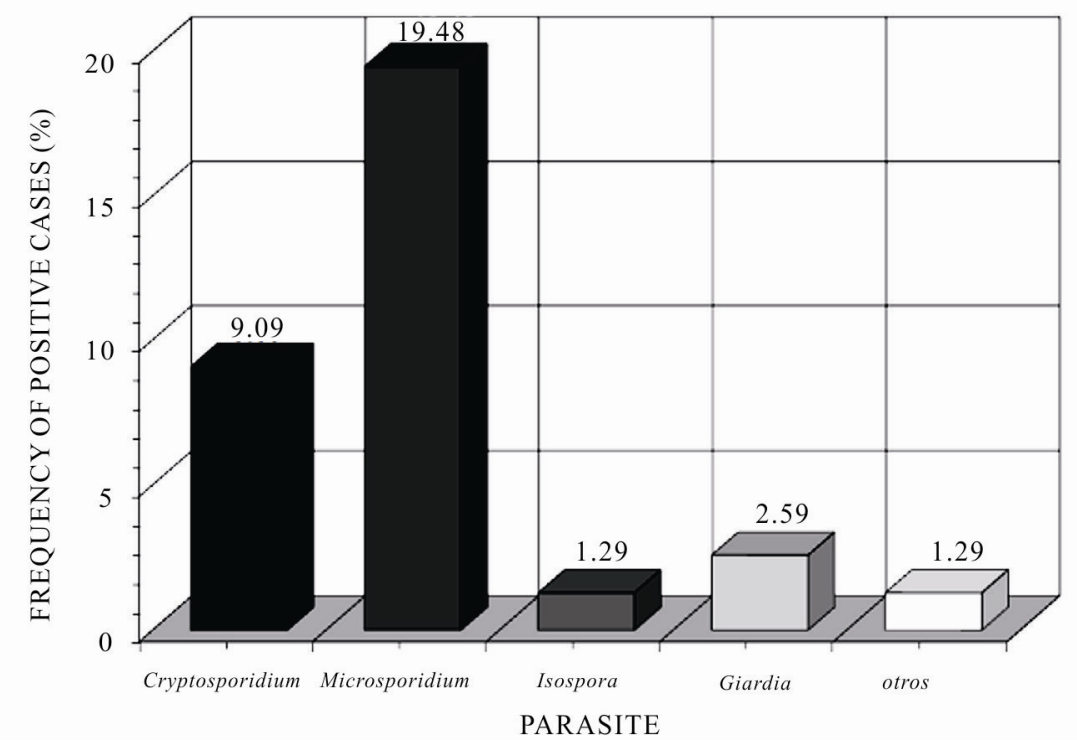

Figure 2. Positive cases of parasites identified in stool from HIV patients from Clinic specializated Condesa (a) and in feces from oncological patients from Children Hospital of Mexico (b).

the length of disease.

Estimates of Cryptosporidium spp. [28,29] frequency in HIV patients have ranged from $16 \%-20 \%$ around the world [30,31]. In our study, the Faust staining method had the highest diagnostic efficiency for Cryptosporidium spp. of the three staining methods, but was less efficient than the molecular PCR analysis. The frequency of Microsporidium spp. was higher in children with an oncological diagnosis, presumably due to the detrimental effects of chemotherapy on their immune systems. However, parasite infections do not always manifest symptoms in children, which is a distinction from adults. The data from our study agree with previous reports of Microsporidium spp. cases accounting for $10 \%-27 \%$ of
HIV patients $[32,33]$. The exceedingly small size of Microsporidia spores $(1-3 \mu \mathrm{M})$ requires light microscopy with oil immersion and staining, which complicates diagnosis based solely upon microscopic analysis and can lead to false negative results. The frequency of Isospora spp. in HIV patients in the US and Europe has been reported as $15 \%$ and $18.2 \%$, respectively $[34,35]$. For Isospora spp., however, the probability of obtaining a false negative result with traditional light microscopy is minimal due to the large size of the unsporulated oocysts that are readily visible even without staining (Figure 1). Giardia spp. have been reported in $8 \%-50 \%$ of HIV patients worldwide [36], while the rates of Cyclospora spp. reported among HIV patients worldwide have been 
(a)

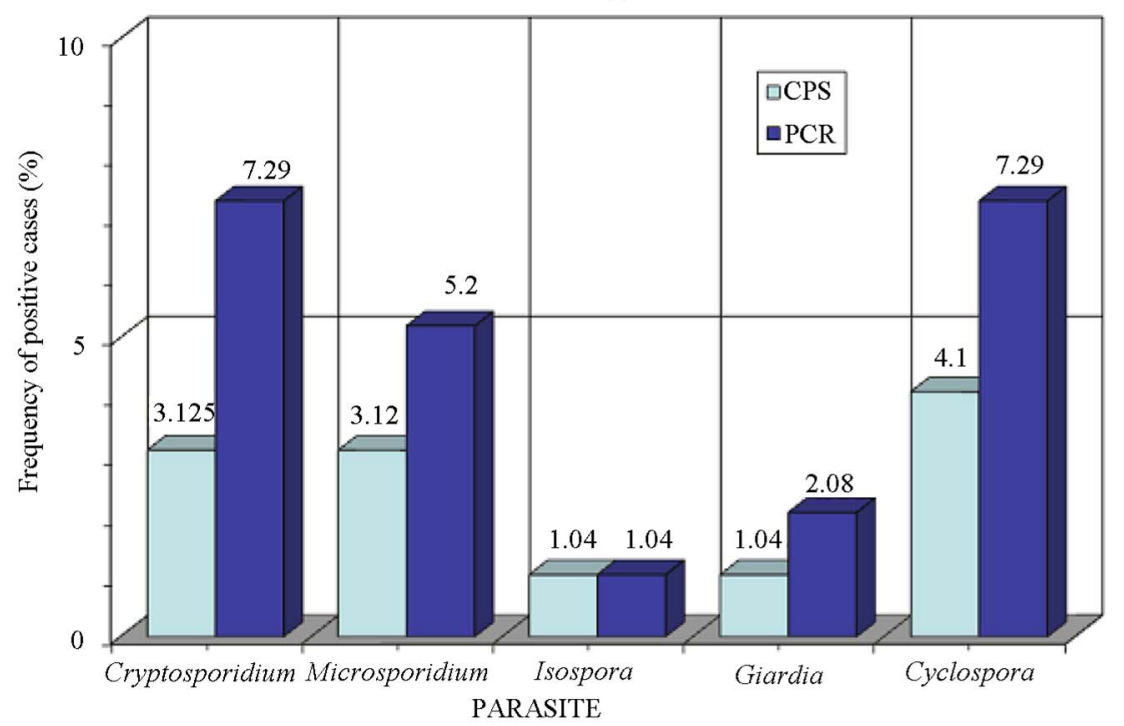

(b)

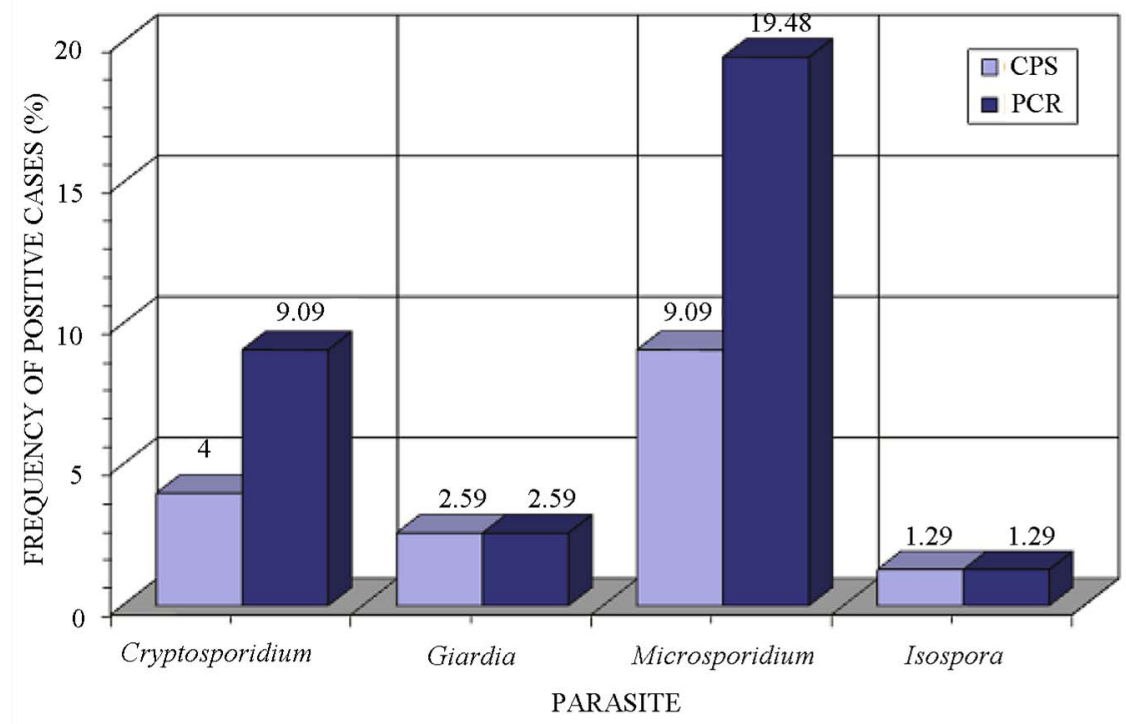

Figure 3. Differences between the parasites identification by coprological analysis plus Ziehl Neelsen staining method and PCR amplification of HIV patients from Clinic specializated Condesa (a) and ALL patients from Children Hospital of Mexico (b).

relatively low (3\% - 4.5\%) [37], the frequency in our HIV study population was even lower. In general, identification of Cyclospora spp. has been based on microscopic observation of the oocyst, but this parasite does not stain with Lugol's iodine, making diagnosis reliant on the results from the other two staining methods only. An accurate molecular approach, such as PCR, will especially benefit diagnosis of this particular parasitic infection. In our study, $100 \%$ of the samples determined as C. cayetanensis-positive by PCR were also positive by the staining method.

To date, only two studies in the literature have re- ported on the incidence of emerging parasites in stool samples of patients with ALL or another kind of cancer. In those, Microsporidia spp. [38] was reported in 1.8\% of patients and Cryptosporidium species [39] in 6.3\%. The mere fact of such limited data availability suggests that the problem of emerging parasites in oncological groups is underestimated globally.

Since prevalence data for the emerging parasites examined in our study are currently not available for the country-wide population of Mexico, the results presented herein provide novel and important insights into the current profile of emerging parasites in immunosuppressed 


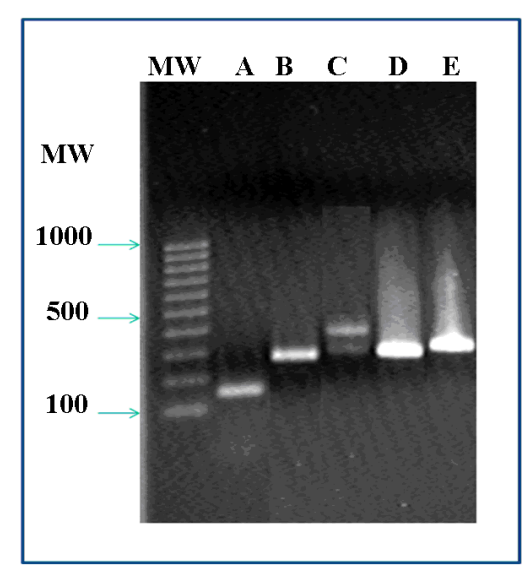

Figure 4. 2\% Agarose gel electrophoresis of PCR stained with ethidium bromide. Specific amplicons of each parasite were obtained according to the conditions early described; they were run simultaneously with molecular weight (MW) 100 bp ladder. (A) Cryptosporidium spp.; an amplicon of $180 \mathrm{bp}$ is observed. (B) A band of 300 bp corresponding to Microsporidium spp. (C) The amplification of Isospora belli give a 400 bp fragment. (D) Giardia intestinalis amplified a fragment of $298 \mathrm{bp}$ and (E) Nested-PCR of Cyclospora cayetanensis allowed obtaining a 300 bp fragment.

patients from a large metropolitan area of Mexico (Mexico City). The presence, frequency, and distribution of opportunistic or emerging parasites in patients with a deficient immune system is very important, as it impacts not only the quality of life of these patients (if manifested as chronic diarrhea) but may also have effects on the disease course, therapeutic efficacy and tolerability, and mortality. Improved diagnostic methods for these parasites will feature higher specificity and sensitivity to facilitate more accurate and earlier diagnosis, so that the appropriate therapeutic intervention may be initiated in a timely manner.

\section{REFERENCES}

[1] K. N. Prasad, V. L. Nag, T. N. Dhole and A. Ayyagari, "Identification of Enteric Pathogens in HIV-Positive Patients with Diarrhoea in Northern India," Journal of Health, Population and Nutrition, Vol. 18, No. 1, 2000, pp. 23-26.

[2] S. Gupta, S. Narang, V. Nunavath and S. Singh, "Chronic Diarrhoea in HIV Patients: Prevalence of Coccidian Parasites," Indian Journal of Medical Microbiology, Vol. 26, No. 2, 2008, pp. 172-175. doi:10.4103/0255-0857.40536

[3] N. A. Foudraine, G. J. Weverling, T. van Gool, M. T. Roos, F. de Wolf, P. Koopmans, et al., "Improvement of Chronic Diarrhea in Patients with Advanced HIV-1 Infection during Potent Antiretroviral Therapy," AIDS, Vol. 12, No. 1, 1998, pp. 35-41.

[4] T. Flanigan, C. Whalen and J. Turner, "Cryptosporidium Infection and CD4 Counts," Annals of Internal Medicine,
Vol. 116, No. 10, 1992, pp. 840-842. doi:10.7326/0003-4819-116-10-840

[5] P. Viriyavejakul, R. Nintasen, C. Punsawad, U. Chaisri, B. Punpoowong and M. Riganti, "High Prevalence of Microsporidium Infection in HIV-Infected Patients," The Southeast Asian Journal of Tropical Medicine and Public Health, Vol. 40, No. 2, 2009, pp. 223-228.

[6] K. Wasson and R. L. Peper, "Mammalian Microsporidiosis," Veterinary Pathology, Vol. 37, No. 2, 2002, pp. 113128. doi:10.1354/vp.37-2-113

[7] R. Bialek, N. Binder, K. Dietz, J. Knobloch and U. E. Zelck, "Comparison of Autofluorescence and Iodine Staining for Detection of $I$. belli in Feces," The American Journal of Tropical Medicine and Hygiene, Vol. 67, No. 3, 2002, pp. 304-305.

[8] R. D. Adam, "Biology of Giardia lamblia," Clinical Microbiology Reviews, Vol. 14, No. 3, 2001, pp. 447-475. doi:10.1128/CMR.14.3.447-475.2001

[9] I. Asma, S. Johari, B. L. Sim and Y. A. Lim, "How Common Is Intestinal Parasitism in HIV-Infected Patients in Malaysia?" Tropical Biomedicine, Vol. 28, No. 2, 2011, pp. 400-410.

[10] R. C. Thompson and A. Smith, "Zoonotic Enteric Protozoa," Veterinary Parasitology, Vol. 182, No. 1, 2011, pp. 70-78. doi:10.1016/j.vetpar.2011.07.016

[11] P. A. Orlandi, L. Carter, A. M. Brinker, A. J. da Silva, D. M. Chu, K. A. Lampel, et al., "Targeting Single-Nucleotide Polymorphisms in the $18 \mathrm{~S}$ rRNA Gene to Differentiate Cyclospora Species from Eimeria Species by Multiplex PCR," Applied and Environmental Microbiology, Vol. 69, No. 8, 2003, pp. 4806-4813.

doi:10.1128/AEM.69.8.4806-4813.2003

[12] J. C. Weitz, C. R. Weitz, M. R. Canales and R. R. Moya, Cyclospora cayetanensis Infection: Updated Review a Propos of Three Cases of Traveler's Diarrhea," Revista Chilena de Infectología, Vol. 26, No. 6, 2009, pp. 549554.

[13] J. M. Shields and B. H. Olson, "PCR-Restriction Fragment Length Polymorphism Method for Detection of Cyclospora cayetanensis in Environmental Waters without Microscopic Confirmation," Applied and Environmental Microbiology, Vol. 69, No. 8, 2003, pp. $4662-$ 4669. doi:10.1128/AEM.69.8.4662-4669.2003

[14] Y. R. Ortega and R. Sanchez, "Update on Cyclospora cayetanensis, a Food-Borne and Waterborne Parasite," Clinical Microbiology Reviews, Vol. 23, No. 1, 2010, pp. 218-234. doi:10.1128/CMR.00026-09

[15] D. S. Linday, J. P. Dubey and B. L. Blagburn, "Biology of Isospora spp. from Humans, Nonhuman Primates, and Domestic Animals," Clinical Microbiology Reviews, Vol. 10, No. 1, 1997, pp. 19-34.

[16] H. F. Tian, B. Chen and J. F. Wen, "Giardiasis, Drug Resistance, and New Target Discovery," Infectious Disorders_Drug Targets, Vol. 10, No. 4, 2010, pp. 295-302. doi:10.2174/187152610791591629

[17] E. S. Didier and L. M. Weiss, "Microsporidiosis: Current Status," Current Opinion in Infectious Diseases, Vol. 19, 
No. 5, 2006, pp. 485-492.

doi:10.1097/01.qco.0000244055.46382.23

[18] A. B. Di Gliullo, M. S. Cribari, A. J. Bava, J. S. Cicconetti and R. Collazos, "Cyclospora cayetanensis in Sputum and Stool Samples," Revista do Instituto de Medicina Tropical de São Paulo, Vol. 42, No. 2, 2000, pp. 115-117. doi:10.1590/S0036-46652000000200009

[19] T. Geurden, D. Berkvens, S. Casaert, J. Vercruysse and E. Claerebout, "A Bayesian Evaluation of Three Diagnostic Assays for the Detection of Giardia duodenalis in Symptomatic and Asymptomatic Dogs," Veterinary Parasitology, Vol. 157, No. 1-2, 2008, pp. 14-20.

doi:10.1016/j.vetpar.2008.07.002

[20] C. M. Coyle, M. Wittner, D. P. Kotler, C. Noyer, J. M. Orenstein, H. B. Tanowitz, et al., "Prevalence of Microsporidiosis Due to Enterocytozoon bieneusi and Encephalitozoon (Septata) intestinalis among Patients with AIDSRelated Diarrhea: Determination by Polymerase Chain Reaction to the Microsporidian Small-Subunit rRNA Gene," Clinical Infectious Diseases, Vol. 23, No. 5, 1996, pp. 1002-1006. doi:10.1093/clinids/23.5.1002

[21] E. C. Faust, J. S. D’Antoni, V. Odom, M. J. Miller, C. Peres, W. Sawitz, et al., "A Critical Study of Clinical Laboratory Techniques for the Diagnosis of Protozoan Cysts and Helminth Eggs in Feces," The American Journal of Tropical Medicine and Hygiene, Vol. 18, 1938, pp. 169-183.

[22] D. Botero and M. Restrepo, "Parasitosis Humanas," Corporación Para Investigaciones Biológicas, Medellín, 2007.

[23] N. Jothikumar, A. J. da Silva, I. Moura, Y. Qvarnstrom and V. R. Hill, "Detection and Differentiation of Cryptosporidium hominis and Cryptosporidium parvum by Dual TaqMan Assays," Journal of Medical Microbiology, Vol. 57, No. 9, 2008, pp. 1099-1105. doi:10.1099/jmm.0.2008/001461-0

[24] N. Chabchoub, R. Abdelmalek, F. Mellouli, F. Kanoun, M. Thellier, A. Bouratbine, et al., "Genetic Identification of intestInal microsporidia Species in Immunocompromised Patients in Tunisia," The American Journal of Tropical Medicine and Hygiene, Vol. 80, No. 1, 2009, pp. 24-27.

[25] S. Jongwutiwes, C. Putaporntip, M. Charoenkorn, T. Iwasaki and T. Endo, "Morphologic and Molecular Characterization of Isospora belli Oocysts from Patients in Thailand," The American Journal of Tropical Medicine and Hygiene, Vol. 77, No. 1, 2007, pp. 107-112.

[26] H. Van Keulen, W. L. Homan, S. L. Erlandsen and E. Jarroll, "A Three-Nucleotide Signature Sequence in Small Subunit rRNA Divides Human Giardia in Two Different Genotypes," Journal of Eukaryotic Microbiology, Vol. 42, No. 4, 1995, pp. 392-394. doi:10.1111/j.1550-7408.1995.tb01600.x

[27] J. R. Yu and W. M. Sohn, "A Case of Human Cyclosporiasis Causing Traveler's Diarrhea after Visiting Indonesia," Journal of Korean Medical Science, Vol. 18, No. 5, 2003, pp. 738-741.

[28] F. J. Enriquez, C. R. Avila, J. I. Santos, J. Tanaka-Kido, O. Vallejo and C. R. Sterling, "Cryptosporidium Infec- tions in Mexican Children: Clinical, Nutritional, Enteropathogenic, and Diagnostic Evaluations," The American Journal of Tropical Medicine and Hygiene, Vol. 56, No. 3, 1997, pp. 254-257.

[29] P. Morán, A. Gómez, A. Valadez, G. García, F. Ramos, E. González, et al., "Periodicity and Patterns of Entamoeba histolytica and E. dispar Infection in HIV+/AIDS Patients in Mexico," Annals of Tropical Medicine and Parasitology, Vol. 103, No. 4, 2009, pp. 307-315. doi:10.1179/136485909X435030

[30] R. A. Dillingham, R. Pinkerton, P. Leger, P. Severe, R. L. Guerrant, J. W. Pape, et al., "High Early Mortality in Patients with Chronic Acquired Immunodeficiency Syndrome Diarrhea Initiating Antiretroviral Therapy in Haiti: A Case-Control Study," The American Journal of Tropical Medicine and Hygiene, Vol. 80, No. 6, 2009, pp. 1060-1064.

[31] S. D. Patel, D. M. Kinariwala and T. B. Javadekar "Clinico-Microbiological Study of Opportunistic Infection in HIV Seropositive Patients," Indian Journal of Sexually Transmitted Diseases, Vol. 32, No. 2, 2011, pp. 90-93. doi:10.4103/0253-7184.85411

[32] A. Espern, F. Morio, M. Miegeville, H. Illa, M. Abdoulaye, V. Meyssonnier, et al., "Molecular Study of Microsporidiosis Due to Enterocytozoon bieneusi and Encephalitozoon intestinalis among Human Immunodeficiency Virus-Infected Patients from Two Geographical Areas: Niamey, Niger, and Hanoi, Vietnam," Journal of Clinical Microbiology, Vol. 45, No. 9, 2007, pp. 2999-3002. doi:10.1128/JCM.00684-07

[33] L. Tuli, A. K. Gulati, S. Sundar and T. M. Mohapatra, "Correlation between CD4 Counts of HIV Patients and Enteric Protozoan in Different Seasons-An Experience of a Tertiary Care Hospital in Varanasi (India)," BMC Gastroenterology, Vol. 8, 2008, p. 36. doi:10.1186/1471-230X-8-36

[34] M. Lagrange-Xélot, R. Porcher, C. Sarfati, N. de Castro, O. Carel, J. D. Magnier, et al., "Isosporiasis in Patients with HIV Infection in the Highly Active Antiretroviral Therapy Era in France," HIV Medicine, Vol. 9, No. 2, 2008, pp. 126-130. doi:10.1111/j.1468-1293.2007.00530.x

[35] O. P. Neira, M. E. Barthel, L. G. Wilson and S. N. Muñoz, "Isospora belli Infection in HIV Positive Patients: Report of 2 Cases and Literature Review," Revista Chilena de Infectología, Vol. 27, No. 3, 2010, pp. 219-227.

[36] A. Lono, S. Kumar and T. T. Chye, "Detection of microsporidia in Local HIV-Positive Population in Malaysia," Transactions of the Royal Society of Tropical Medicine and Hygiene, Vol. 105, No. 7, 2011, pp. 409-413. doi:10.1016/j.trstmh.2011.03.006

[37] A. Kurniawan, T. Karyadi, S. W. Dwintasari, I. P. Sari, E. Yunihastuti, S. Djauzi and H. V. Smith, "Intestinal Parasitic Infections in HIV/AIDS Patients Presenting with Diarrhoea in Jakarta, Indonesia," Transactions of the Royal Society of Tropical Medicine and Hygiene, Vol. 103, No. 9, 2009, pp. 892-898. doi:10.1016/j.trstmh.2009.02.017

[38] J. H. Botero, A. Castaño, M. N. Montoya, N. E. Ocampo, 

by Coprological and Molecular Analysis

M. I. Hurtado and M. M. Lopera, “A Preliminary Study of the Prevalence of Intestinal Parasites in Immunocompromised Patients with and without Gastrointestinal Manifestations," Revista do Instituto de Medicina Tropical de São Paulo, Vol. 45, No. 4, 2003, pp. 197-200. doi:10.1590/S0036-46652003000400004
[39] H. Nahrevanian and M. Assmar, "Cryptosporidiosis in Immunocompromised Patients in the Islamic Republic of Iran," Journal of Microbiology, Immunology and Infection, Vol. 41, No. 1, 2008, pp. 74-77. 